\title{
No Scalar Hair Theorem for a Charged Spherical Black Hole
}

\author{
N.Banerjee* and S.Sen** \\ Relativity and Cosmology Research Centre, \\ Department of Physics, Jadavpur University, \\ Calcutta-700032, India.
}

\begin{abstract}
This paper consolidates the no scalar hair theorem for a charged spherically symmetric black hole in four dimensions in general relativity as well as in all scalar tensor theories, both minimally and nonminimally coupled, when the effective Newtonian constant of gravity is positive. However, there is an exception when the matter field itself is coupled to the scalar field, such as in dilaton gravity.
\end{abstract}

*narayan@juphys.ernet.in

**somasri@juphys.ernet.in 


\section{Introduction}

Very recently Saa ${ }^{1}$ deduced a theorem which shows that the static spherically symmetric exterior solutions for the gravitational field equations in a very wide class of scalar tensor theories will essentially reduce to the wellknown Schwarzschild solutions if one has to hide the essential singularity at the centre of symmetry by an event horizon. The no-hair conjecture in general relativity says that in the exterior of a black hole, the only information available regarding the black hole may be that of its mass, charge and angular momentum. For an uncharged spherical black hole, therefore, the only information available to an external observer is about its mass. Saa ${ }^{1}$ used the solution for the uncharged spherically symmetric mass distribution along with a minimally coupled scalar field as discussed extensively by Xanthopoulos and Zannias $^{2}$. It is readily found that the scalar field becomes trivial if one demands a regular horizon at a finite distance from the centre of symmetry. This is in perfect agreement with the no-hair conjecture. The importance of Saa's work lies in the fact that by virtue of a class of transformations and choice of functions, he could generate the corresponding static spherically symmetric exterior solutions in a large number of scalar tensor theories and thus could show the validity of "no scalar hair theorem" in these theories. The scalar field in these theories becomes a constant and thus trivial if one demands a blackhole i.e, a well defined event horizon shielding the singularity at the centre.

In the case of a spherically symmetric system with an electromagnetic field, the same result is expected, i.e, for the existence of an event horizon, the scalar field is likely to become trivial. But this conclusion cannot be taken for granted because of the nonlinearity of the gravitational field equations.

In the present work, we make an attempt to generalise Saa's work to include an electromagnetic field. We start with the action for a very general scalar tensor theory of gravity coupled with a Maxwell field,

$$
S\left[g_{\mu \nu}, \phi, F_{\mu \nu}\right]=\int\left[f(\phi) R-h(\phi) g^{\mu \nu} \phi_{, \mu} \phi_{, \nu}-F_{\mu \nu} F^{\mu \nu}\right] \sqrt{-g} d^{4} x
$$

where $g_{\mu \nu}, \phi$ and $F_{\mu \nu}$ are the metric tensor, the scalar field, and the Maxwell field respectively, $f(\phi)$ and $h(\phi)$ are positive functions of the scalar field. It should be noted that the scalar field is nonminimally coupled to gravity and as a result the 
Newtonian constant $\mathrm{G}$ becomes a function of $\phi$. This action in special cases reduces to the corresponding actions for Brans-Dicke ${ }^{4}$ theory or the generalized scalar tensor theory of Nordtvedt ${ }^{5}$. For Brans-Dicke theory $f(\phi)=\phi$ and $h(\phi)=\frac{\omega}{\phi}$ where $\omega$ is a constant parameter. If $\omega$ is a function of $\phi$ instead of being constant with the same choice for $f(\phi)$ and $h(\phi)$, the action corresponds to that for Nordtvedt's generalised scalar tensor theory. For $f(\phi)=1-\frac{1}{6} \phi^{2}$ and $h(\phi)=\frac{1}{2}$ we get the conformally coupled scalar field of Bekenstein ${ }^{3}$.

In the present work we show that by using a conformal transformation of the metric tensor and redefining the scalar field in a similar way as discussed by Saa, the nonminimal coupling between the geometry and the scalar field can be broken and the action would correspond to that of an Einstein-Maxwell field along with a zero mass scalar meson field. The corresponding solutions for a static spherically symmetric spacetime had been given by Penney ${ }^{6}$ way back in 1969 . We show that in Penney's solution the scalar field becomes trivial if one demands a regular horizon. We use the inverse transformation of $g_{\mu \nu}$ to get corresponding solutions in a broad class of nonminimally coupled theories in order to investigate the conditions for a black hole to exist. The result is the same, the scalar field becomes trivial when we demand that an event horizon should exist. It deserves mention, however, that the function $f(\phi)$ is restricted to positive values only.

In the case of a conformally coupled scalar field, where $f(\phi)=\left(1-\frac{\phi^{2}}{6}\right)$, Bekenstein ${ }^{3}$ obtained a black hole solution but indicated it is not a genuine black hole as the scalar field diverges at the event horizon. In a subsequent paper $^{7}$, however, he proved that there is no geodetic incompleteness in the motion of a test particle and the tidal forces are all regular even in the nontrivial presence of the scalar field and concluded that this example indeed represents a genuine black hole. Hence this gives a counter example to the no scalar hair theorem. The value of $f(\phi)$, in this case is negative near the event horizon. Very recently Mayo and Bekenstein ${ }^{8}$ considered the no scalar hair theorem extensively for a multiplet of minimally coupled scalar fields as well as for a non-minimally coupled scalar field where the coupling with the Ricci scalar in the lagrangian is given by

$$
f(\phi)=1-\chi \phi^{2} .
$$

They proved the non existence of the scalar hair in such cases for a general value of 
$\chi$, namely $\chi<0$ or $\chi>\frac{1}{2}$. The work of Mayo and Bekenstein is very powerful as it takes into account self interacting scalar fields, i.e, nonlinear potential functions in the action.

The case of a multiplet of scalar fields had, infact, been discussed previously by Bekenstein $^{9}$. In this work Bekenstein also extended his work to some nonminimally coupled generalised scalar tensor theory ${ }^{5}$ for a conformally transformed version of the theory ( see Dicke ${ }^{10}$ ).

Although the present work does not discuss the inclusion of potentials, it widens the scalar no hair theorem in the sense that it is valid not only in general relativity but practically in all scalr tensor theories for $f>0$ with the known exception of dilaton gravity.

In next section, the conformal transformation which breaks the nonminimal coupling between geometry and scalar field is discussed and the corresponding static solutions for a charged sphere with a minimally coupled scalar field are given. Section 3 deals with some examples of nonminimally coupled scalar tensor theories and in section 4 a brief discussion on the already known results regarding charged black holes in dilaton gravity is given. In section 5 we include a discussion on the results obtained.

\section{Charged spherical black holes with a minimally coupled scalar field}

The coupled Einstein-Maxwell-Scalar field equations, obtained by varying the action (1.1) with respect to $g_{\mu \nu}, \phi$ and the Maxwell potential $\mathrm{A}^{\mu}$, are given by

$$
\begin{gathered}
f(\phi) R_{\mu \nu}-h(\phi) \phi_{, \mu} \phi_{, \nu}-\left\{f(\phi)_{, \mu}\right\}_{; \nu}-\frac{1}{2} g_{\mu \nu} \square f(\phi)=\left(T_{\mu \nu}-\frac{1}{2} T g_{\mu \nu}\right), \\
2 h(\phi) \square \phi-h^{\prime}(\phi) g^{\mu \nu} \phi_{, \mu} \phi_{, \nu}+f^{\prime}(\phi) R=0,
\end{gathered}
$$

and

$$
F_{; \nu}^{\mu \nu}=0
$$


respectively, where units are so chosen that $8 \pi \mathrm{G}$ and $\mathrm{c}$ are unity. $\mathrm{T}_{\mu \nu}$ represents the energy momentum tensor for the electromagnetic field, given by

$$
T_{\mu \nu}=g^{\alpha \beta} F_{\alpha \mu} F_{\beta \nu}-\frac{1}{4} g_{\mu \nu} F_{\alpha \beta} F^{\alpha \beta}
$$

For such a field, $\mathrm{T}$, the trace of $\mathrm{T}_{\mu \nu}$ is zero. By virtue of the Bianchi identity, the wave equation for the scalar field, (2.2), is not an independent equation, but rather follows from the other field equations.

With a conformal transformation of the form

$$
g_{\mu \nu}=\Omega^{2} \bar{g}_{\mu \nu}
$$

the Ricci scalar $R$ transforms as ${ }^{11}$

$$
R=\Omega^{-2} \bar{R}-6 \Omega^{-3} \square \Omega
$$

An overhead bar indicates that the variables are in the transformed version. If we choose

$$
\Omega^{-2}=f(\phi)
$$

and redefine the scalar field as $\bar{\phi}$, given by,

$$
\bar{\phi}(\phi)=\sqrt{2} \int_{\phi_{0}}^{\phi} d \xi \sqrt{\frac{3}{2}\left(\frac{d}{d \xi} \ln f(\xi)\right)^{2}+\frac{h(\xi)}{f(\xi)}},
$$

the action (1.1) becomes

$$
\bar{S}\left[\bar{g}_{\mu \nu}, \bar{\phi}, \bar{F}_{\mu \nu}\right]=\int\left[\bar{R}-\frac{1}{2} \bar{g}_{\mu \nu} \bar{\phi}_{, \mu} \bar{\phi}_{, \nu}-\bar{F}^{2}\right] \sqrt{-\bar{g}} d^{4} x .
$$

This result is obtained for any arbitrary positive definite lower limit " $\phi_{0}$ " in equation (2.8). This method is exactly similar to the one discussed by Saa, the only difference being, in that work the electromagnetic field had not been considered. It is easy to check that

$$
\sqrt{-g} F^{2}=\sqrt{-g} F_{\mu \nu} F^{\mu \nu}=\sqrt{-\bar{g}} \bar{F}_{\mu \nu} \bar{F}^{\mu \nu}=\sqrt{-\bar{g}} \bar{F}^{2}
$$

The field equations now become formally similar to those for a coupled EinsteinMaxwell system along with a minimally coupled scalar field.The equations look like

$$
\bar{G}_{\mu \nu}=\bar{\phi}_{, \mu} \bar{\phi}_{, \nu}-\frac{1}{2} \bar{g}_{\mu \nu} \bar{\phi}^{, \alpha} \bar{\phi}_{, \alpha}-\bar{g}^{\alpha \beta} \bar{F}_{\alpha \mu} \bar{F}_{\beta \nu}+\frac{1}{4} \bar{g}_{\mu \nu} \bar{F}_{\alpha \beta} \bar{F}^{\alpha \beta}
$$




$$
\left(\bar{F}^{\mu \nu}\right)_{; \nu}=0
$$

and

$$
\square \bar{\phi}=0 .
$$

The solutions for this set of equations had been given by Penney ${ }^{6}$. The line element for the static spherically symmetric spacetime is written as

$$
d s^{2}=-e^{\bar{\gamma}} d t^{2}+e^{\bar{\alpha}} d r^{2}+e^{\bar{\beta}}\left(d \theta^{2}+\sin \theta^{2} d \phi^{2}\right)
$$

In the coordinates where $\bar{\alpha}+\bar{\gamma}=0$, the solutions are

$$
\begin{gathered}
e^{\bar{\alpha}}=e^{-\bar{\gamma}}=(r-a)^{-\Lambda}(r-b)^{-\Lambda}\left[\frac{b(r-a)^{\Lambda}-a(r-b)^{\Lambda}}{(b-a)}\right]^{2}, \\
e^{\bar{\beta}}=(r-a)^{1-\Lambda}(r-b)^{1-\Lambda}\left[\frac{b(r-a)^{\Lambda}-a(r-b)^{\Lambda}}{(b-a)}\right]^{2}, \\
\bar{\phi}=\frac{c}{a-b} \ln \frac{r-a}{r-b}=\sqrt{\frac{1-\Lambda^{2}}{2}} \ln \left(\frac{r-a}{r-b}\right)
\end{gathered}
$$

and

$$
\bar{F}_{14}=q e^{-\bar{\beta}}
$$

The constants $\Lambda, a, b, c$ are related by the equations

$$
\begin{gathered}
2 \Lambda^{2} a b=q^{2}, \\
a+b=2 m, \\
\Lambda^{2} c^{2}=\left(1-\Lambda^{2}\right)\left(2 \Lambda^{2} m^{2}-q^{2}\right),
\end{gathered}
$$

where $m$ and $q$ are recognised to be the total mass and charge of the static sphere. The constant of integration $c$ is actually a sort of scalar charge. The constant $\Lambda$ can take values between 0 and 1 . For $\Lambda=1, c$ is zero and the scalar field becomes trivial and equations (2.15) and (2.16) yield the usual Reissner -Nordstrom(RN) solutions. For $\mathrm{q}=0$, one gets the solution for a static sphere with a minimally coupled scalar field.

The Ricci scalar $R$, for the spacetime given by equations (2.15) and (2.16), looks like

$$
R=\frac{(a-b)^{2}}{2(r-a)^{2-\Lambda}(r-b)^{2-\Lambda}} \frac{\left(1-\Lambda^{2}\right)}{\left[b(r-a)^{\Lambda}-a(r-b)^{\Lambda}\right]^{2}} .
$$


This expression shows that the solution is asymptotically flat. From the metric tensor components, one finds that, $r=a$ and $r=b$ are coordinate singularities. It is evident from equation (2.20) that if $\Lambda \neq 1$, for both the surfaces $r=a$ and $r=b$ the Ricci scalar blows up, whereas when $\Lambda=1$ for both these surfaces,

$$
R=0
$$

and these surfaces act as event horizons to shield the essential singularity at $r=0$. Again in this case, i.e for $\Lambda=1$, the scalar field $\bar{\phi}$ becomes trivial and the line element given by equations (2.15) and (2.16) reduces to the wellknown Reissner-Nordstrom solution. So, with a scalar meson field, one can conclude that the only charged spherical black hole that exists is the RN black hole.

With the help of the transformations (2.5) and (2.7), one can generate the corresponding solutions for different nonminimally coupled scalar tensor theories, and check whether a black hole other than RN one is possible or not. As the transformation equation suggests it is apperent that any spherical black hole given by action (1.1) will be RN one. However, in the next section, we shall discuss some specific examples.

\section{Charged black holes with nonminimally coupled Scalar Fields}

\section{Case I: Brans-Dicke Theory}

If one makes the choice

$$
f(\phi)=\phi,
$$

and

$$
h(\phi)=\frac{\omega}{\phi}
$$

where $\omega$ is a dimensionless constant parameter, the action (1.1) reduces to the one in Brans-Dicke theory. With the help of equation (2.8), one can write

$$
\bar{\phi}(\phi)=\sqrt{(2 \omega+3)} \ln \frac{\phi}{\phi_{0}},
$$


which, when compared with (2.17), yields

$$
\phi=\phi_{0}\left(\frac{r-a}{r-b}\right)^{\sqrt{\left(1-\Lambda^{2}\right) / 2(2 \omega+3)}} .
$$

By using the inverse of the transformation equation (2.5) one can now write the metric in Brans-Dicke theory as

$$
\begin{gathered}
\phi_{0} d s^{2}=\left(\frac{r-b}{r-a}\right)^{\sqrt{\left(1-\Lambda^{2}\right) / 2(2 \omega+3)}}\left[-(r-a)^{\Lambda}(r-b)^{\Lambda}\left(\frac{b(r-a)^{\Lambda}-a(r-b)^{\Lambda}}{(b-a)}\right)^{-2} d t^{2}\right. \\
+(r-a)^{-\Lambda}(r-b)^{-\Lambda}\left(\frac{b(r-a)^{\Lambda}-a(r-b)^{\Lambda}}{(b-a)}\right)^{2} d r^{2} \\
\left.+(r-a)^{1-\Lambda}(r-b)^{1-\Lambda}\left(\frac{b(r-a)^{\Lambda}-a(r-b)^{\Lambda}}{(b-a)}\right)^{2}\left(d \theta^{2}+\sin ^{2} \theta d \phi^{2}\right)\right]
\end{gathered}
$$

and the Ricci scalar $R=R_{\mu}^{\mu}$ as

$$
\begin{gathered}
R=\frac{\omega}{\phi^{2}} g^{\mu \nu} \phi_{, \mu} \phi_{, \nu} \\
=\frac{\left(1-\Lambda^{2}\right) \omega \phi_{0}(a-b)^{4}}{2(2 \omega+3)} \frac{\left[b(r-a)^{\Lambda}-a(r-b)^{\Lambda}\right]^{-2}}{(r-a)^{2-\Lambda-p}(r-b)^{2+p-\Lambda}}
\end{gathered}
$$

where

$$
p=\sqrt{\frac{\left(1-\Lambda^{2}\right)}{2(2 \omega+3)}} .
$$

Now one has a singularity at $r=0$. For $\Lambda \neq 1, r=a$ or $r=b$ surfaces also have curvature singularities as evident from the metric (3.4) and the expression for the Ricci scalar $R$. For $\Lambda=1$, however, $r=a$ and $r=b$ are not curvature singularities and act as event horizons. But in this case, i.e, for $\Lambda=1$, the scalar field becomes trivial, $\phi=\phi_{0}$, and the theory reduces to the Einstein-Maxwell system.

\section{Case II: Generalised Scalar Tensor Theories}

If $\omega=\omega(\phi)$, instead of being a constant, we get a generalisation of Brans-Dicke theory. This type of theory had been proposed by Bergman ${ }^{12}$, Wagoner ${ }^{13}$, and Nordtvedt $^{5}$. Different choices of $\omega$ as a function of $\phi$ can be made depending on the particular physical interest. The action (1.1) reduces to that for Nordvedt's theory if

$$
f(\phi)=\phi
$$


and

$$
h(\phi)=\frac{\omega(\phi)}{\phi}
$$

In what follows, we shall take up two choices of $\omega(\phi)$ in order to verify the no scalar hair theorem.

\section{(i) Barker's choice}

In this choice ${ }^{14}$,

$$
\omega(\phi)=\frac{4-3 \phi}{2(\phi-1)}
$$

Equation (2.8) yields, on integration, the result

$$
\bar{\phi}=\arctan \frac{\sqrt{\phi-1}-\sqrt{\phi_{0}-1}}{1+\sqrt{(\phi-1)\left(\phi_{0}-1\right)}},
$$

which, in view of equation (2.17) yields

$$
\phi=\sec ^{2}\left[\arctan \sqrt{\phi_{0}-1}+\sqrt{\frac{1-\Lambda^{2}}{2}} \ln \frac{r-a}{r-b}\right] .
$$

With the help of equations (2.15), (2.16), (2.5), (2.7) and (3.9), one can write the metric as

$$
\begin{aligned}
& d s^{2}= \cos ^{2}\left[\arctan \sqrt{\phi_{0}-1}+\sqrt{\frac{1-\Lambda^{2}}{2}} \ln \frac{r-a}{r-b}\right] \\
& {\left[-(r-a)^{\Lambda}(r-b)^{\Lambda}\left[\frac{b(r-a)^{\Lambda}-a(r-b)^{\Lambda}}{(b-a)}\right]^{-2} d t^{2}\right.} \\
&+(r-a)^{-\Lambda}(r-b)^{-\Lambda}\left[\frac{b(r-a)^{\Lambda}-a(r-b)^{\Lambda}}{(b-a)}\right]^{2} d r^{2} \\
&\left.+(r-a)^{1-\Lambda}(r-b)^{1-\Lambda}\left[\frac{b(r-a)^{\Lambda}-a(r-b)^{\Lambda}}{(b-a)}\right]^{2}\left(d \theta^{2}+\sin ^{2} \theta d \phi^{2}\right)\right],
\end{aligned}
$$

and the expression for $R$ becomes

$$
\begin{gathered}
R=\frac{\omega}{\phi^{2}} g^{\mu \nu} \phi_{, \mu} \phi, \nu-\frac{3}{\phi} \frac{\omega^{\prime}}{2 \omega+3} g^{\mu \nu} \phi_{, \mu} \phi_{, \nu} \\
=\frac{4\left(1-\Lambda^{2}\right)(a-b)^{4}}{(r-a)^{2-\Lambda}(r-b)^{2-\Lambda}} \frac{\sec ^{2}\left[\arctan \sqrt{\phi_{0}-1}+\sqrt{\frac{1-\Lambda^{2}}{2}} \ln \frac{r-a}{r-b}\right]}{\left\{b(r-a)^{\Lambda}-a(r-b)^{\Lambda}\right\}^{2}} .
\end{gathered}
$$




\section{(ii) Schwinger's choice}

This case is given by ${ }^{15}$

$$
2 \omega+3=\frac{1}{\alpha \phi},
$$

$\alpha$ being a constant. Following the same procedure as in the Brans-Dicke or Barker's theory we get the solution for the scalar field in Schwinger's theory as

$$
\phi=\left[\frac{1}{\sqrt{\phi_{0}}}-\sqrt{\frac{\left(1-\Lambda^{2}\right) \alpha}{8}} \ln \frac{r-a}{r-b}\right]^{-2}
$$

and the line element as

$$
\begin{gathered}
d s^{2}=\left[\frac{1}{\sqrt{\phi_{0}}}-\sqrt{\frac{\left(1-\Lambda^{2}\right) \alpha}{8}} \ln \frac{r-a}{r-b}\right]^{2} \\
{\left[-(r-a)^{\Lambda}(r-b)^{\Lambda}\left[\frac{b(r-a)^{\Lambda}-a(r-b)^{\Lambda}}{(b-a)}\right]^{-2} d t^{2}\right.} \\
+(r-a)^{-\Lambda}(r-b)^{-\Lambda}\left[\frac{b(r-a)^{\Lambda}-a(r-b)^{\Lambda}}{(b-a)}\right]^{2} d r^{2} \\
\left.+(r-a)^{1-\Lambda}(r-b)^{1-\Lambda}\left[\frac{b(r-a)^{\Lambda}-a(r-b)^{\Lambda}}{(b-a)}\right]^{2}\left(d \theta^{2}+\sin ^{2} \theta d \phi^{2}\right)\right],
\end{gathered}
$$

and the Ricci Scalar $R$ is given by

$$
\begin{array}{r}
R=\frac{1-\Lambda^{2}}{4} \frac{(a-b)^{4}}{\{(r-a)(r-b)\}^{2-\Lambda}}\left\{b(r-a)^{\Lambda}-a(r-b)^{\Lambda}\right\}^{-2} \\
{\left[\frac{1}{\sqrt{\phi_{0}}}-\sqrt{\frac{\left(1-\Lambda^{2}\right) \alpha}{8}} \ln \frac{r-a}{r-b}\right]^{-2} .}
\end{array}
$$

In both these subcases, namely (i) Barker's theory and (ii) Schwinger's theory, the solutions and the Ricci scalar reveal that $r=a$ or $r=b$ are event horizons only if $\Lambda=1$. If $\Lambda \neq 1$, these surfaces are singular by themselves and therefore there will be naked singularities and no black holes. Moreover, for $\Lambda=1$, the solutions in both these cases reduce to the usual RN solution as $\phi$ becomes trivial. 


\section{Spherical charged black holes in dilaton gravity}

In the weak field limit of the string theory, one obtains Einstein gravity along with a nonminimally coupled scalar field, called the dilaton field. The action in dilaton gravity is

$$
S=\int d^{4} x \sqrt{-g}\left[e^{-2 \phi} R+4 e^{-2 \phi} g^{\mu \nu} \phi_{, \mu} \phi_{, \nu}-e^{2 \alpha \phi} F^{\mu \nu} F_{\mu \nu}\right]
$$

which is different from the action (1.1) as the matter lagrangian (here the electromag-

netic field, given by $L_{e m}=-F_{\mu \nu} F^{\mu \nu}$ ) also is coupled to the scalar field. In context of (1.1), action (4.1) can be rewritten as

$$
S=\int d^{4} x \sqrt{-g}\left[f(\phi) R-h(\phi) g^{\mu \nu} \phi_{, \mu} \phi_{, \nu}-k(\phi) F^{\mu \nu} F_{\mu \nu}\right]
$$

where $k(\phi)$ takes care of the coupling between the scalar field and the matter field. This coupling is retained even under the conformal transformation of the form (2.5) and (2.7). The transformed action looks like

$$
\bar{S}=\int d^{4} x \sqrt{-\bar{g}}\left[\bar{R}-\frac{1}{2} \bar{g}^{\mu \nu} \bar{\phi}_{, \mu} \bar{\phi}_{, \nu}-\bar{k}(\bar{\phi}) \bar{F}^{\mu \nu} \bar{F}_{\mu \nu}\right]
$$

where $\bar{k}(\bar{\phi})=k(\phi(\bar{\phi}))$. The coupling between the scalar field and the Maxwell field is broken only if $k(\phi)=1$. The static spherically symmetric solutions for the field equations relevant to the action (4.3) shows that the asymptotic behaviour of the black hole is characterised by the mass, the electric charge and a dilatonic charge (ref. 16 and 17). The survival of this dilatonic charge does not jeopardise the existence of a well defined horizon and hence a black hole. So the no scalar hair theorem is not applicable in the case of dilaton gravity. In the special case when $k(\phi)=1$, however, the scalar field becomes trivial if one insists on the existence of an event horizon. These features of dilaton black holes have been discussed by Garfinkle et al ${ }^{16}$ and Rakhmanov ${ }^{17}$ amongst others. We also refer to the work of Horowitz and Strominger ${ }^{18}$ where the existence of blackholes had been discussed in higher dimensions in string theory as well as in p-branes.

\section{Conclusion}

In sections 2 and 3 it is shown that the charged static spherically symmetric asymptotically flat solutions for the field equations lead to black holes only if the scalar field 
becomes trivial both in cases of minimal and nonminimal coupling of the scalar field. This result crucially depends on the assumption that the coupling function $f(\phi)$ in equation (1.1) is positive. A negative $f(\phi)$, in the weak field limit, however, gives rise to a negative Newtonian constant G. The work of Mayo and Bekenstein includes such couplings also although for a limited kind of $f(\phi)\left[f(\phi)=1-\chi \phi^{2}\right]$. With a necessarily positive effective Newtonian constant of gravity $[f(\phi)>0]$ the present work enlarges the domain of applicability of the scalar no hair theorem to a wide class of scalar tensor theories of gravity.

An important point to note is that for any dimension $\mathrm{n}$ other than $n=4$, a non trivial coupling between the scalar field and the Maxwell field exist in the transformed action after the conformal transformation in different general scalar tensor theories also and thus for higher ( or lower) dimensional theories the black holes are different from black holes having no scalar hair in four dimension. This difference between $n=4$ and $n \neq 4$ arises because equation (2.10) is valid only for $n=4$. A recent work of Cai and Myung ${ }^{19}$ in Brans-Dicke theory can be referred to in this context.

These results consolidates the no scalar hair theorem, proved earlier by Bekenstein and Mayo-Bekenstein in a different approach for a charged spherical black hole in four dimensions for a positive definite $f(\phi)$ when the electromagnetic field itself is not coupled to the scalar field.

It remains to be seen, however, whether this remains valid along with scalar potentials in an arbitrary scalar tensor theory. The case of a negative $f(\phi)$ also deserves extensive investigation in a wider class of scalar tensor theories particularly in view of the results obtained by Bekenstein ${ }^{7}$. It will also be important to investigate the stability of the black hole solution, if any, in such cases. For a discussion in detail on this point we refer to the work of Mayo and Bekenstein ${ }^{8}$.

\section{Acknowledgement}

One of the authors (S.S) is thankful to University Grants' Commission of India for the financial support. 


\section{References}

${ }^{1}$ A.Saa, J.Math.Phys, 37, 2346, (1996)

${ }^{2}$ B,C.Xanthopoulos and T.Zannias, Phys.Rev.D, 40, 2564, (1989)

${ }^{3}$ J.D.Bekenstein, Ann.Phys., 82, 535, (1974)

${ }^{4}$ C.Brans and R.H.Dicke, Phys.Rev, 124, 925, (1961)

${ }^{5}$ K.Nordtvedt Jr. Astrophys.J, 161, 1059, (1970)

${ }^{6}$ R.Penney, Phys.Rev, 182,1383, (1969)

${ }^{7}$ J.D.Bekenstein, Ann.Phys., 91, 75 (1975)

${ }^{8}$ A.E.Mayo and J.D.Bekenstein, Phys.RevD, 54, 5059, (1996)

${ }^{9}$ J.D.Bekenstein, Phys.Rev.D, 51, R6608, (1995)

${ }^{10}$ R.H.Dicke, Phys.Rev, 125, 2163, (1962)

${ }^{11}$ J.L.Synge, Relativity: The General Theory (North Holland

Publishing Co. (1960))

${ }^{12}$ P.G.Bergman, Int.J.Theor.Phys., 1, 25 (1968)

${ }^{13}$ R.V.Wagoner, Phys.Rev.D, 1, 3209, (1970)

${ }^{14}$ B.M.Barker, Astrophys.J., 219, 5, (1978)

${ }^{15}$ J.Schwinger, Particles, Sources and Fields (Addison-Wesley, Reading,Massachusetts, (1970))

${ }^{16}$ D.Garfinkle, G.T.Horowitz and A.Strominger, Phys.Rev.D, 43, 3140 (1991)

${ }^{17}$ M.Rakhmanov, Phys.RevD, 50, 5155, (1994)

${ }^{18}$ G.T.Horowitz and A.Strominger, Nucl.Phys.B, 360, 197, (1991)

${ }^{19}$ R.G.Cai and Y.S.Myung, Phys.Rev.D, 56, 3466, (1997) 